\title{
Adjective Agreement in Noon: Evidence for a Split Theory of Noun-Modifier Concord
}

\author{
Nico Baier
}

LSA Annual Meeting, Portland, January 8-11, 2015

Main Claim. In this paper, I show that two distinct mechanisms are needed to account for the pattern of noun-adjective agreement found in Noon (Cangin, Senegal). I argue that one form of agreement on adjectives is derived by a valuation of syntactic $\varphi$-probe on $\mathrm{A}^{0}$, while another is derived via a post-syntactic process of feature copying. This lends support to the idea that the narrow syntactic operation Agree does not always coincide with morphological agreement (Chung To Appear).

Background. Much previous work presupposes that the same mechanism underlies noun-modifier agreement (often called concord) and argument predicate agreement (see, e.g. Baker 2008; Carstens 2013; Danon 2011; Toosarvandani and van Urk 2012). T hese accounts assume that the same operation underlies both types of agreement: usually a modified version of Agree (Chomsky 2001). An alternative approach, advocated by Norris (2014), argues that noun-modifier agreement cannot be unified with argument-predicate agreement. Norris proposes that noun-modifier agreement occurs in the morphology. He adopts a Distributed Morphology account in which an operation of Feature Copying values AGR-nodes which are inserted into the structure post-syntactically (Noyer 1997; Kramer 2010).

Adjective Agreement in Noon. Adjectives in Noon exhibit two types of agreement. First, adjectives take a prefix that encodes the class and number of the noun they modify. This prefix is obligatory in both predicative contexts, (1a), and attributive contexts, (1b):

$$
\begin{aligned}
& \text { a. baay } \varnothing \quad \text { fi } \text {-yak } \\
& \operatorname{dog}(\mathrm{C} 2 \mathrm{~s}) \text { COP C2S-big } \\
& \text { 'A dog is big.' }
\end{aligned}
$$
b. [DP baay [i -yak ] $\operatorname{dog}(\mathrm{C} 2 \mathrm{~s})$ C2s-big

Second, attributive adjectives must agree in definiteness with the noun they modify. Definiteness is marked by a suffix on the head noun that reflects the its class/number along with three degrees of deixis. Adjectives must take an identical suffix, as shown by (2b). Predicative adjectives cannot take a definite suffix, even when the subject is definite, as shown by (2a).
a. baay-faa
$\varnothing \quad$ fi-yak- $(*$ faa $)$ dog-DEF:C2S COP C2s-big 'the dog (over there) is big.'
b. [DP baay-faa dog-DEF:C2s C2s-big
'The big dog (over there).'

The core observation is that prefixal agreement and definiteness agreement have different distributions: prefixal agreement is required in both attributive and predicative contexts, but definiteness agreement is only available for attributive adjectives (that is, DP-internally).

Assumptions. I assume that Noon DPs have the basic structure in (3). Attributive adjectives (AP) are adjoined to NP. The head-initial order of the Noon DP is derived via head movement of $\mathrm{N}^{0}$ to $\mathrm{D}^{0}$ via $\mathrm{Num}^{0}$. Following Danon (2011), I assume class features originate on $\mathrm{N}^{0}$ and number features on $\mathrm{Num}^{0}$, but that the result of head movements is a single $\varphi$-bundle at the complex $\mathrm{D}^{0}$ :

$$
\left[\mathrm{DP} N+\mathrm{Num}+\mathrm{D}_{[\varphi: v a l]}[\mathrm{NumP} \mathrm{Num}[\mathrm{NP} \mathrm{AP}[\mathrm{NP} \mathrm{N}]]]\right]
$$

The Noon DP 
I assume adjectival predicates are formed via a head Pred $^{0}$ which takes an AP as its complement and the subject in its specifier, as shown in (4)

(4) $\left[\right.$ PreP DP $\left.\operatorname{DUBJ}_{\text {SU }}[\operatorname{Pred}[\mathrm{AP}]]\right]$

Adjectival Predicates

The Problem. A unified approach to noun-modifier agreement fails to account for the assymetry between attributive and predicative contexts observed above. Under a purely syntactic account, the head $\mathrm{A}^{0}$ would be merged with two probes: one with unvalued $\varphi$-features (the prefix) and with unvalued $\varphi$-features and an unvalued definiteness-feature (the suffix). Such an account would then have to stipulate why both probes can be valued in attributive contexts, but only the prefixrelated probe can find a value in the predicative contexts. A post-syntactic account faces a similar conundrum. In this type of account, both morphemes would be associated with their own AGRnode. Again, however, one would have to stipulate why the feature copying operation responsible for valuing these AGR-nodes is unable to value the suffixal node in predicative contexts.

Analysis. I propose that the two different agreement morphemes on Noon adjectives derive from two distinct agreement mechanisms. Concretely, I argue that prefixal agreement spells out a $\varphi$ probe on $\mathrm{A}^{0}$. Following Carstens (2013) and Toosarvandani and van Urk (2012), I assume a probe may be valued via Agree by a c-commanding head once it has unsuccessfully probed into its own c-command domain. $\mathrm{A}^{0}$ is merged with unvalued $\varphi$-features, at which point these features probe and fail to find a suitable goal. In predicative contexts, $\mathrm{A}^{0}$ 's probe finds matching features when the c-commanding subject DP is merged in Spec-PredP, as shown in (5). In attributive contexts, $\mathrm{A}^{0}$ 's features are valued by the head created by movement of $\mathrm{N}^{0}$ to $\mathrm{D}^{0}$.

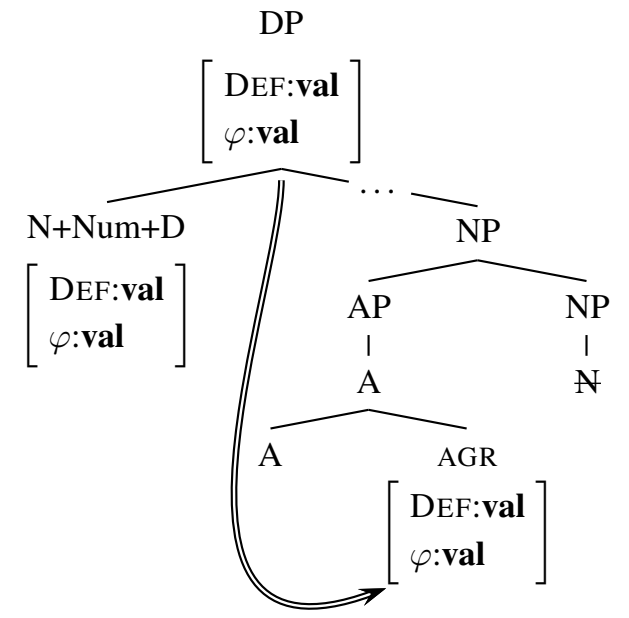

Figure 1: Feature Copying to AGR on A

Definiteness agreement is post-syntactic. Following Norris (2014), I assume that post-syntactic agreement involves the insertion of an AGR-node on the specified head. This AGR-node is subsequently valued via a Feature Copying operation. Valuation of an AGR-node by Feature Copying is dependent on a different structural relation than Agree. While Agree is sensitive to c-command, Feature Copying is sensitive to dominance: features are copied to the AGR-node from the closest dominating projection with suitable features. After Norris (2014), I assume there is a feature percolation process in the syntax by which features from the complex head $[\mathrm{N}+\mathrm{Num}+\mathrm{D}]$ percolate 
to the DP-phrase level so that they can be accessible outside the DP. In attributive contexts, the AGR-node on $\mathrm{A}^{0}$ is valued by the features of DP, as shown in (6). In predicative contexts, however, the requisite dominance relation does not hold between the subject DP and $\mathrm{A}^{0}$. Therefore, valuation of any AGR-node inserted on a predicative adjective is impossible, as Feature Copying cannot proceed. I argue that this is what blocks the insertion of the AGR-node. In other words, AGR-nodes are only inserted when there are features able to be copied to them. Thus, AGR-nodes such as the definiteness agreement suffix in Noon will only be available to DP-internal, i.e. attributive, adjectives.

Extensions. The analysis developed here can also be extended to a well known difference between Romance and West Germanic. Romance adjectives agree in both attributive and predicative contexts, whereas adjectives in West Germanic only agree in attributive contexts (Vikner 2001). Under the present analysis, this means that $\mathrm{A}^{0}$ is always merged with unvalued $\varphi$-features in Romance, while in West Germanic, an AGR-node is inserted onto $\mathrm{A}^{0}$ only in attributive contexts. Further support for this conclusion comes from the fact that West Germanic adjective agreement is sensitive to definiteness, just like post-syntactic agreement in Noon.

More generally, it seems that post-syntactic agreement of the sort hypothesized above can involve a wider array of features than narrow syntactic agreement. While definiteness agreement occurs in attributive contexts in several languages (Kramer 2010), I know of no language where it occurs with adjectival predicates. Positing a difference between two distinct agreement mechanisms moves us toward an explanation of this assymetry.

Conclusion. Noun-adjective in Noon agreement provides evidence for two distinct mechanisms underlying agreement: one syntactic and one morphological. This supports the idea that Agree does not always coincide with morphological agreement.

\section{References}

Baker, Mark C. 2008. On the Nature of the Antiagreement Effect: Evidence from Wh-in-situ in Ibibio. Linguistic Inquiry 39:615-632.

Carstens, Vicki. 2013. Delayed valuation: a reanalysis of "upwards" complementizer agreement and the mechanics of case. MS., University of Missouri.

Chomsky, Noam. 2001. Derivation by phase. In Ken Hale: A life in linguistics, ed. Michael Kenstowicz, 1-52. Cambridge, Massachusetts: MIT Press.

Chung, Sandra. To Appear. On reaching agreement late. In CLS 48: Papers from the parases- sions.

Danon, Gabi. 2011. Agreement and DP-internal feature distribution. Syntax 14:297-317.

Kramer, Ruth. 2010. The Amharic definite marker and the syntax-morphology interface. Syntax 13:196240.

Norris, Mark. 2014. A theory of nominal concord. Doctoral Dissertation, UC Santa Cruz.

Noyer, Rolf. 1997. Features, positions and affixes in autonomous morphological structure. Garland Publishing.

Toosarvandani, Maziar, and Coppe van Urk. 2012. Directionality and intervention in nominal concord: Evidence from zazaki ezafe. Handout version of a poster at NELS 43, CUNY.

Vikner, Sten. 2001. Predicative adjective agreement, volume Sprachkontakt, Sprachvergleich, Sprachvariation: Festschrift für Gottfriede Kolde zum 65. 